\title{
Childhood intellectual disability and parents' mental health: integrating social, psychological and genetic influences
}

Kate Baker, Rory T. Devine, Elise Ng-Cordell, F. Lucy Raymond, IMAGINE-ID consortium* and Claire Hughes

\section{Background}

Intellectual disability has a complex effect on the well-being of affected individuals and their families. Previous research has identified multiple risk and protective factors for parental mental health, including socioeconomic circumstances and child behaviour.

\section{Aims}

This study explored whether genetic cause of childhood intellectual disability contributes to parental well-being.

\section{Method}

Children from across the UK with intellectual disability due to diverse genetic causes were recruited to the IMAGINE-ID study. Primary carers completed the Development and Well-being Assessment, including a measure of parental distress (Everyday Feeling Questionnaire). Genetic diagnoses were broadly categorised into aneuploidy, chromosomal rearrangements, copy number variants (CNVs) and single nucleotide variants.

\section{Results}

Compared with the UK general population, IMAGINE-ID parents $(n=888)$ reported significantly elevated emotional distress (Cohen's $d=0.546$ ). Within-sample variation was related to recent life events and the perceived impact of children's difficulties. Impact was predicted by child age, physical disability, autistic characteristics and other behavioural difficulties. Genetic diagnosis also predicted impact, indirectly influencing parental well-being. Specifically, CNVs were associated with higher impact, not explained by CNV inheritance, neighbourhood deprivation or family structure.

\section{Conclusions}

The mental health of parents caring for a child with intellectual disability is influenced by child and family factors, converging on parental appraisal of impact. We found that genetic aetiologies, broadly categorised, also influence impact and thereby family risks. Recognition of these risk factors could improve access to support for parents, reduce their long-term mental health needs and improve well-being of individuals with intellectual disability.

\section{Declaration of interest}

None.

\section{Keywords}

Intellectual disability; genetics; carers; depressive disorders; risk assessment.

\section{Copyright and usage}

(c) Royal College of Psychiatrists 2020. This is an Open Access article, distributed under the terms of the Creative Commons Attribution licence (http://creativecommons.org/licenses/by/4.0/), which permits unrestricted re-use, distribution, and reproduction in any medium, provided the original work is properly cited.

\section{Parental mental health and childhood intellectual disability}

It is estimated that between 1 in 50 and 1 in 20 families worldwide include at least one child with intellectual disability, defined as significant and persistent impairments in cognitive ability and adaptive function. ${ }^{1}$ Carer mental health risks are underrecognised, compounding family stress and entailing long-term consequences for well-being of the whole family. ${ }^{2}$ Mothers and fathers caring for a child with intellectual disability are at risk of psychiatric disorders including depression and anxiety. ${ }^{3-5}$ More recent accounts have shifted from a sole focus on adverse effects of childhood disability to a recognition that families have capacity for adaptation and resilience as well as risk, and that family experiences are diverse. ${ }^{6}$ Sociocultural factors, child characteristics and parent characteristics may each contribute to this diversity. Epidemiological studies of families affected by intellectual disability in the UK have consistently highlighted the importance of social context for parental experience. Lone-parent households and families facing financial challenges are especially likely to include a child with intellectual disability, and socioeconomic indices can account for 50\% of the elevated risk of poor self-esteem and self-efficacy in mothers. ${ }^{7}$ Similarly, matching for socioeconomic variables in the

* See Supplementary material, available at https://doi.org/10.1192/bjp 2020.38, for consortium membership and affiliations.
Millennium Cohort Study attenuated (but did not abolish) elevated psychiatric risk in fathers and mothers of young children with cognitive delay. ${ }^{5}$ Parental physical health and psychological resources may also be important determinants of risk and resilience. ${ }^{7,8}$ Prior level of depression is a key predictor of later depression within parents of children with intellectual disability; ${ }^{9}$ conversely, positive parental characteristics, such as dispositional optimism, locus of control and sense of coherence, may help maintain well-being. ${ }^{10,11}$ Other studies highlight the heterogeneity of childhood neurodevelopmental phenotypes: salient features relevant to parental well-being include age and developmental stage, with adolescence identified as a particularly risky period; ${ }^{12}$ presence of physical disability and illness; autism spectrum characteristics and affective disturbance or conduct problems. ${ }^{13}$ Indeed, longitudinal studies have shown bidirectional relationships between child behaviour and parenting stress, which may be a magnification of transactional dynamics observed for children without intellectual disability. ${ }^{14}$

\section{The contribution of intellectual disability aetiology to family risks}

In high-income countries, it is estimated that at least $60 \%$ of severe intellectual disability is associated with specific, identifiable genetic variants, encompassing large chromosomal abnormalities (i.e., 
aneuploidy, translocations), submicroscopic deletions and duplications (i.e. copy number variants; CNVs) and DNA sequence differences (i.e. single nucleotide variants; SNVs). ${ }^{15}$ The implementation of genome-wide technologies (chromosomal microarrays, wholeexome or whole-genome sequencing) is leading to a rapid increase in the proportion of children with a developmental disorder receiving a genetic diagnosis, i.e. identification of a genetic variant that either causes or contributes to intellectual disability. ${ }^{16}$ This provides new opportunities to investigate the heterogeneity of intellectual disability at all levels, from molecular mechanisms to social dynamics. One relatively unexplored question concerns the potential influence of intellectual disability aetiology on family outcomes and parental well-being. ${ }^{17}$ Early studies reported better well-being in mothers of children with Down syndrome compared with mothers of children with autism or idiopathic developmental delay, although group differences attenuated over time and when contrasts in social and communicative abilities were taken into account. ${ }^{18}$ In a study of 150 children with fragile X syndrome and their families, behavioural problems of children with, and siblings without fragile X syndrome both predicted maternal distress, suggesting that child behaviour exerts a powerful influence within these families. ${ }^{19}$ In a recent study, Cornelia de Lange syndrome, Smith Magenis syndrome and 1p36 deletion syndrome were associated with elevated risk of maternal depression, irrespective of the age or ability level of diagnosed children, whereas these background factors were the major determinants of maternal mental health for ten other genetic conditions. ${ }^{20}$ Potential explanations for these associations include physiological or behavioural phenotypes (e.g. sleep disturbance, volatility or anxiety), or societal factors (e.g. variation in understanding and acceptance of different diagnoses). Turning to 'new' genetic diagnoses, CNVs in particular may be relevant to understanding family risks. A high proportion of these intellectual disability-associated variants are inherited from parents, ${ }^{21}$ who may or may not have neurodevelopmental difficulties themselves. CNVs are associated with lifespan mental health difficulties, thus potentially amplifying risks in both the parent and child. ${ }^{22}$ Rather than directly affecting parental well-being, genetic diagnosis is likely to act indirectly on parental well-being via its influence on children's functional outcomes and families' experiences.

\section{Aims}

This study appraised the importance of genetic diagnoses, broadly categorised, alongside other risk factors for carer mental health in the context of childhood intellectual disability. Building on the results of previous studies (notably the study by Emerson; ${ }^{4}$ see Supplementary Table 1), we assessed the influence of child characteristics, social factors and parental psychological appraisal (impact), and explored the direct and indirect effects of genetic diagnosis.

\section{Method}

\section{Recruitment}

The authors assert that all procedures contributing to this work comply with the ethical standards of the relevant national and institutional committees on human experimentation and with the Helsinki Declaration of 1975, as revised in 2008. All procedures were approved by London-Queen Square Research Ethics Committee (14/LO/1069).

Participants were recruited to the Intellectual Disability and Mental Health: Assessing the Genomic Impact on Neurodevelopment (IMAGINE-ID) study between 2014 and 2018. Recruitment to the study was by referral from any of the 22
UK regional genetics centres (75.5\%), patient support groups (11.2\%), other research projects (3.6\%) and self-referrals via social media and online advertising (9.7\%). At the time of recruitment to the study most families (92\%) resided in England, with $4.3 \%$ residing in Scotland, 3.2\% in Wales and $0.1 \%$ in Northern Ireland. The entry criteria for referral of an index child to the study were as follows: clinically documented developmental delay or intellectual disability, a molecular genetic diagnosis documented from an accredited diagnostic laboratory and at least 3 years of age at recruitment. Children meeting these entry criteria were identified retrospectively from regional genetics centres and research databases and prospectively in out-patient clinics. Parents or guardians were sent an invitation letter, enabling them to contact the study, provide consent and participate. Written informed consent was obtained from parents or guardians of all child participants aged $<16$ years, and an assent form was completed by the child where possible. Data presented in this paper were collected from a parent or carer of the index child via online data entry $(78 \%)$, online with telephone support (5.2\%), telephone interview (14.9\%) or face-to-face interview (1.8\%), according to parent/carer choice.

Diagnostic genetic reports were obtained from medical records or directly from families. Variants were broadly categorised as per Table 1, which describes frequency of genetic diagnosis types and variant inheritance for the sample. Where multiple pathogenic or likely pathogenic variants had been reported for a participant, categorisation was based on the variant with highest pathogenicity rating according to clinical laboratory report.

\section{Assessments of parental well-being and impact}

The primary respondent completed the ten-item Everyday Feeling Questionnaire $^{23}$ (EFQ) for themselves and for their partner. Items were rated on a 5-point scale ranging from 0 (none of the time) to 4 (all of the time). Items were summed together to create a total score. Higher scores indicated higher levels of psychological distress (Cronbach $\alpha=0.89$ ). The respondent also completed the Impact Supplement of the Strengths and Difficulties Questionnaire (SDQ), ${ }^{24}$ in which they rated the extent to which their child's difficulties caused distress, interfered with their home, school and social lives and were burdensome to the family. Items were summed together to create an impact score, with higher scores indicating a greater level of impact.

\section{Assessments of child characteristics}

The primary respondent completed the Development and Wellbeing Assessment (DAWBA) ${ }^{25}$ structured assessment. This measure has been used in large-scale studies of mental health among children with and without intellectual disability, ${ }^{26,27}$ and its validity when administered online or by telephone has been established. ${ }^{28}$ Developmental quotient was calculated from parental estimation of current mental age divided by chronological age (in lieu of standardised assessments, which were not feasible within the study protocol). The primary respondent provided information within the DAWBA about the presence or absence of physical disabilities in the target child, including difficulties with toileting, speech, vision, hearing, movement and seizures. ${ }^{27}$ The number of disabilities was summed together to create a total score, with higher scores indicating a greater level of physical disability (mean 2.41, s.d. 1.87, ordinal $\alpha=0.79$ ). Children received three scores based on the DAWBA algorithms generating $70 \%$ likelihoods for 12 different psychiatric diagnoses: a binary score indicating whether or not the child had a likely diagnosis of autism spectrum disorder (ASD); a binary score indicating whether or not the child likely had a conduct disorder or oppositional defiant disorder (CD/ODD) and a binary score indicating whether or not the child likely had any other diagnosis. 


\begin{tabular}{|c|c|c|c|c|c|c|c|c|c|c|c|c|c|c|c|c|c|c|}
\hline \multirow{4}{*}{$\begin{array}{l}\text { Inheritance of genetic diagnosis } \\
N\end{array}$} & \multirow{2}{*}{\multicolumn{3}{|c|}{$\begin{array}{l}\text { Whole sample, } \\
\qquad N=888\end{array}$}} & \multicolumn{15}{|c|}{ Genetic diagnosis } \\
\hline & & & & \multicolumn{3}{|c|}{$\begin{array}{l}\text { CNV, } \\
n=541\end{array}$} & \multicolumn{3}{|c|}{$\begin{array}{l}\text { SNV } \\
n=148\end{array}$} & \multicolumn{3}{|c|}{$\begin{array}{l}\text { Multiple CNV } \\
\quad n=119\end{array}$} & \multicolumn{3}{|c|}{$\begin{array}{c}\text { Other } \\
\text { chromosomal, } \\
n=46\end{array}$} & \multicolumn{3}{|c|}{$\begin{array}{c}\text { Sex } \\
\text { chromosome } \\
\text { aneuploidy, } \\
n=34\end{array}$} \\
\hline & DN & I & $\mathbf{U}$ & DN & I & $\mathbf{U}$ & DN & I & $\mathbf{U}$ & DN & I & $\mathbf{U}$ & DN & I & U & DN & I & U \\
\hline & 349 & 245 & 294 & 159 & 167 & 215 & 103 & 18 & 27 & 21 & 59 & 39 & 33 & 1 & 12 & 33 & 0 & 1 \\
\hline Descriptive statistics & Mean & & s.d. & Mean & & s.d. & Mean & & s.d. & Mean & & s.d. & Mean & & s.d. & Mean & & s.d. \\
\hline Age & 8.16 & & 2.89 & 7.89 & & 2.82 & 9.43 & & 2.87 & 8.09 & & 2.99 & 7.91 & & 2.37 & 7.50 & & 3.09 \\
\hline $\mathrm{DQ}(N=888)$ & 55.35 & & 21.36 & 58.02 & & 19.43 & 44.52 & & 24.41 & 56.45 & & 22.05 & 51.05 & & 19.80 & 66.61 & & 14.68 \\
\hline Physical $(N=885)$ & 2.01 & & 1.62 & 1.81 & & 1.52 & 2.91 & & 1.75 & 1.92 & & 1.56 & 2.07 & & 1.75 & 1.56 & & 1.40 \\
\hline Impact $(N=887)$ & 5.51 & & 2.90 & 5.72 & & 2.78 & 5.27 & & 3.24 & 5.40 & & 2.83 & 4.37 & & 2.85 & 5.21 & & 3.23 \\
\hline Life events $(N=884)$ & 0.80 & & 1.03 & 0.81 & & 1.04 & 0.73 & & 1.03 & 0.94 & & 1.13 & 0.67 & & 0.83 & 0.62 & & 0.78 \\
\hline $\operatorname{IMD}(N=805)$ & 5.82 & & 2.73 & 5.65 & & 2.71 & 6.29 & & 2.83 & 5.87 & & 2.71 & 6.27 & & 2.73 & 5.71 & & 2.56 \\
\hline $\mathrm{EFQ}(N=882)$ & 16.58 & & 7.39 & 16.66 & & 7.61 & 16.14 & & 7.45 & 17.03 & & 7.19 & 15.29 & & 6.06 & 17.32 & & 5.78 \\
\hline
\end{tabular}

\section{Assessments of social circumstances}

Children's postcodes were used to obtain the Index of Multiple Deprivation, which provides a measure of relative neighbourhood deprivation based on income, employment, education, health, crime, housing and living environment. ${ }^{29}$ Scores range from 1 (the most deprived decile) to 10 (the least deprived decile in England). The primary respondent completed a Recent Negative Life Events checklist for events that had occurred in the past 12 months, e.g., separation owing to marital difficulties. ${ }^{30}$ The number of events was summed together to create a total ranging from 0 to 7 (ordinal $\alpha=0.70$ ); $49 \%$ of families had experienced 1 or more negative life events in the past 12 months. The respondent also provided information about whether they were solo parents, the number of children living at home and the number of adults living at home (UK Office for National Statistics definitions ${ }^{31}$ ).

\section{Analysis}

The primary analyses were conducted using structural equation modelling in Mplus version 8 for Mac OS (www.statmodel. com). ${ }^{32}$ We used a maximum likelihood estimator with robust standard errors in each of our models to account for the nonnormal distribution of our indicators. ${ }^{33}$ We evaluated model fit using three primary criteria: comparative fit index (CFI) $>0.90$, Tucker-Lewis index (TLI) $>0.90$, root-mean-square error of approximation (RMSEA) $<0.08 .^{34}$ Table 1 shows the extent of missing data on each questionnaire. We used a full information approach under the assumption that data were missing at random so that all families were included. Model parameters and standard errors were estimated in Mplus, using all available data.

\section{Results}

\section{Sample characteristics}

The initial sample comprised 990 children and their parents. After siblings $(n=41)$, children with a developmental quotient of $>85$ $(n=32)$ and children with no known genetic diagnosis $(n=29)$ were removed, the final sample comprised 888 children (505 males), aged $4-15$ years (mean age 8.16 years, s.d. 2.89 ) with a mean developmental quotient of 55.35 (s.d. 21.36). Table 1 shows the descriptive statistics for each of the study measures for the whole sample and by genetic diagnosis. There was a broad representation of socioeconomic circumstances within the cohort, although the distribution was shifted toward affluence relative to expectation for childhood intellectual disability (Supplementary Table 2). Of those respondents $(n=797)$ providing information about current parental relationship status, 135 (16.9\%) were solo parents. The majority of respondents had families with one or two children $(n=591,74.2 \%)$, and one-quarter $(25.8 \%)$ had three or more children $(n=206)$. A fifth $(19.6 \%)$ of the sample met the $70 \%$ likelihood criteria for ASD. A fifth (21.5\%) of the sample met the 70\% likelihood criteria for conduct disorder. Supplementary Table 3 shows the correlations between all study measures.

\section{Caregiver distress: comparison between IMAGINE-ID and UK general population parents}

We compared psychological distress (EFQ scores) in the IMAGINE-ID primary respondents and their partners with primary respondent parents and their partners in the general population. ${ }^{23}$ The comparison data were collected from 5279 mothers and other caregivers of 8- to 19-year-old children as part of the 2007 follow-up of the 2004 UK Office for National Statistics survey of the mental health of children and young people in Great Britain. ${ }^{35}$ Summary statistics for analysis were provided by the first author of ${ }^{25}$ as a personal communication. The IMAGINE-ID primary respondents had higher levels of distress (mean 16.258, s.d. 7.39, 95\% CI 16.09-17.07) than primary respondent parents from the general population $(N=5245$, mean 12.545 , s.d. 6.87 , $95 \%$ CI $12.45-12.64, t(881)=16.205$, Cohen's $d=0.546)$. Likewise, partners were reported to have higher levels of distress (mean 14.18 , s.d. $7.06,95 \%$ CI $13.65-14.71)$ than partners in the general population $(N=3889$, mean 10.894 , s.d. $6.54,95 \%$ CI $10.70-11.09$, $t(689)=12.222$, Cohen's $d=0.465)$. Where identity of the primary respondent was provided, levels of distress were higher in mothers (mean 17.01 , s.d. $7.493, N=543$ ) than in fathers (mean 14.13 , s.d. $6.71, N=48, t(589)=2.58$, mean difference $=2.89$, s.e. $1.119,95 \%$ CI 0.69-5.09, Cohen's $d=0.405$ ).

\section{Model 1: predictors of caregiver distress (whole cohort)}

We regressed caregiver distress (measured by the EFQ) onto child characteristics: age in years, gender $(0=$ male, $1=$ female $)$, developmental quotient, $\operatorname{ASD}(0=$ no, $1=$ yes $), \operatorname{CD} / \mathrm{ODD}(0=$ no, $1=$ yes $)$, other psychiatric disorder $(0=$ no, $1=$ yes $)$, physical disability, SDQ social impact score and four dummy variables representing genetic diagnosis (with CNV as the reference group). The dependent variable was also regressed onto five social circumstances variables: total negative life events in the past 12 months, socioeconomic status measured using the index of multiple deprivation, number of 


\begin{tabular}{|c|c|c|c|c|c|c|c|c|c|c|c|c|}
\hline & \multicolumn{4}{|c|}{ Perceived impact } & \multicolumn{4}{|c|}{ Caregiver Depressive symptoms } & \multicolumn{4}{|c|}{$\begin{array}{c}\text { Indirect effects on caregiver } \\
\text { depressive symptoms via perceived } \\
\text { impact }\end{array}$} \\
\hline & Est. & s.e. & $\beta$ & $P$ & Est. & s.e. & $\beta$ & $P$ & Est. & s.e. & $\beta$ & $P$ \\
\hline \multicolumn{13}{|l|}{ Child characteristics } \\
\hline Age & 0.089 & 0.030 & 0.089 & ** & 0.112 & 0.082 & 0.044 & & & & & \\
\hline Gender & -0.104 & 0.172 & -0.018 & & 0.609 & 0.466 & 0.041 & & & & & \\
\hline Developmental quotient & 0.002 & 0.006 & 0.016 & & 0.006 & 0.014 & 0.017 & & & & & \\
\hline Physical disability & 0.165 & 0.067 & 0.093 & * & 0.139 & 0.175 & 0.030 & & & & & \\
\hline DAWBA ASD & 1.266 & 0.225 & 0.174 & $\star \star \star *$ & 0.682 & 0.701 & 0.037 & & & & & \\
\hline DAWBA CD/ODD & 2.224 & 0.192 & 0.316 & $\star * *$ & 1.596 & 0.712 & 0.089 & * & & & & \\
\hline DAWBA other & 1.396 & 0.186 & 0.209 & $* * *$ & -0.044 & 0.635 & -0.003 & & & & & \\
\hline Perceived impact & & & & & 0.719 & 0.094 & 0.282 & $\star * *$ & & & & \\
\hline \multicolumn{13}{|l|}{ Genetic } \\
\hline CNV versus SNV & -0.608 & 0.258 & -0.078 & * & -0.391 & 0.653 & -0.020 & & -0.437 & 0.197 & -0.022 & * \\
\hline CNV versus multiple CNV & -0.388 & 0.254 & -0.046 & & 0.291 & 0.710 & 0.013 & & -0.279 & 0.185 & -0.013 & \\
\hline CNV versus chromosomal & -0.776 & 0.371 & -0.060 & * & -0.185 & 0.861 & -0.006 & & -0.558 & 0.275 & -0.017 & * \\
\hline CNV versus aneuploidy & -0.549 & 0.395 & -0.037 & & 0.883 & 0.986 & 0.023 & & -0.395 & 0.288 & -0.010 & \\
\hline \multicolumn{13}{|l|}{ Social circumstances } \\
\hline Life events & 0.069 & 0.084 & 0.025 & & 0.987 & 0.235 & 0.138 & $* * *$ & & & & \\
\hline IMD & -0.024 & 0.032 & -0.023 & & 0.089 & 0.086 & 0.033 & & & & & \\
\hline Number of children & 0.036 & 0.209 & 0.006 & & 0.225 & 0.573 & 0.013 & & & & & \\
\hline Number of adults & 1.056 & 0.573 & 0.067 & & 1.867 & 1.105 & 0.047 & & & & & \\
\hline Solo parent & -0.320 & 0.259 & -0.042 & & 0.034 & 0.721 & 0.002 & & & & & \\
\hline
\end{tabular}

children in the household ( $0=1-2$ children, $1=3$ or more), lone parent status $(0=$ no, $1=$ yes $)$ and number of adults in the household ( $0=1-2$ adults, $1=3$ or more). We also regressed the social impact scores from the SDQ onto each of the child predictors and five social circumstances variables. All other predictor variables were permitted to correlate. Table 2 shows the direct and indirect effects on each of the dependent variables in model 1. Figure 1 shows a simplified path diagram for model 1 .

The model provided a good fit to the data $\left(\chi^{2}(67)=104.517\right.$, $P=0.002, \quad$ CFI $=0.960, \quad$ TLI $=0.941, \quad$ RMSEA $=0.025,90 \% \quad$ CI $0.015-0.034)$, and accounted for $27.1 \%$ of the variance in SDQ social impact scores and $15.3 \%$ of the variance in caregiver distress. Older age, the presence of physical disability, ASD, CD/ODD and other psychiatric diagnoses on the DAWBA were uniquely associated with higher levels of perceived impact on children's home, school and social life. Children with SNV diagnosis had lower impact scores than children with CNV diagnosis. Children with other chromosomal diagnoses also had lower impact scores than children with CNV diagnosis. No other genetic contrast was significant. High impact score was associated with high parental distress. Negative life events and the presence of CD/ODD was also associated with high levels of distress in the primary caregiver.

We then examined the indirect effects between genetic diagnosis and caregiver distress via impact scores. There was an indirect effect of SNV diagnosis (relative to $\mathrm{CNV}$ ) on caregiver distress via impact. Likewise, there was an indirect effect of chromosomal abnormalities (relative to $\mathrm{CNV}$ ) on caregiver distress via impact. Specifically, relative to having CNV, having SNV or chromosomal diagnoses was linked with lower levels of perceived impact and, in turn, lower levels of caregiver distress.

\section{Model 2: predictors of caregiver distress (CNV diagnoses only)}

In our second model we examined the relations between $\mathrm{CNV}$ characteristics and caregiver distress in those families of children with CNV diagnoses only $(n=541)$. This model was identical to the previous model but included two dummy variables representing inheritance patterns (i.e. de novo $(n=159)$ versus familial inheritance $(n=162)$; and de novo versus unknown inheritance $(n=212))$, two dummy variables indicating the type of CNV (i.e. deletion $(n=338)$ versus duplication $(n=183)$; and deletion versus other $(n=20))$ and a continuous variable indicating $\mathrm{CNV}$ size (mean $2.396 \times 10^{6}$ base pairs, s.d. $3.446 \times 10^{6}$ ). The model provided an acceptable fit to the data $\left(\chi^{2}(74)=91.585, P=0.0810\right.$, $\mathrm{CFI}=0.957, \mathrm{TLI}=0.939, \mathrm{RMSEA}=0.022,95 \%$ CI $0-0.036)$ and accounted for $24.7 \%$ of the variance in SDQ impact scores and $13.8 \%$ of the variance in caregiver distress. The main results are displayed in Table 3. Of note, there were no direct or indirect effects of any of the $\mathrm{CNV}$ characteristics on psychological distress in the primary caregiver or perceived impact.

\section{Discussion}

This study represents the largest investigation to date of the wellbeing of caregivers in the context of childhood intellectual disability, and the first to include evaluation of genetic diagnosis as a potential predictor of risk. In keeping with previous studies, there is a broad distribution of parental emotional well-being, but this distribution is very significantly shifted toward elevated risk in parents caring for a child with intellectual disability. Our results also replicate Emerson's ${ }^{4}$ finding that the social and psychological impact of the child's disability predict parental well-being. Moreover, across both studies, four aspects of a child's intellectual disability-associated phenotypes (i.e. ASD, CD/ODD, additional psychopathology, physical disability), rather than the severity of developmental delay predicted impact scores. The current study identifies genetic diagnosis as an additional predictor of parental well-being, mediated by the perceived impact of a child's difficulties on home, school and social life.

\section{Impact or symptom severity?}

Impact plays an important role within clinical decision making (e.g. in the DSM-5), but there remains debate regarding its measurement 


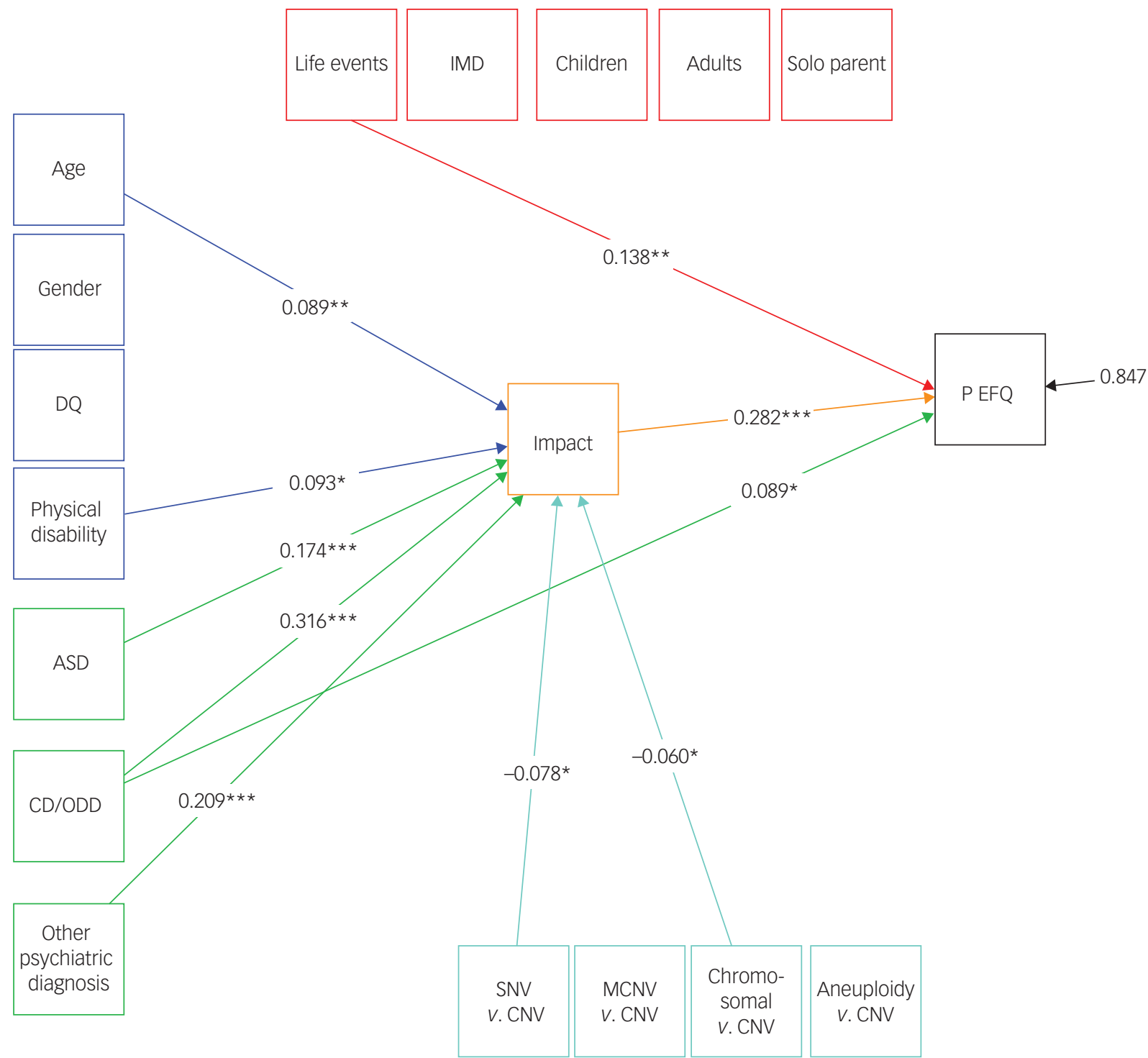

Fig. 1 Model 1 simplified path diagram. Only statistically significant paths are shown. All estimates are standardized robust maximum likelihood estimates.

ASD, autism spectrum disorder; CD/ODD, conduct disorder/oppositional defiant disorder; DQ, developmental quotient; CNV, copy number variant; MCNV, multiple copy number variant; P EFQ, primary respondent Everyday Feelings Questionnaire; IMD, Index of Multiple Deprivation; SNV, single nucleotide variant; Children, number of children in household; Adults, number of adults in household.

$* * * P<0.001, * * P<0.01, * P<0.05$

and interpretation. One perspective is that behavioural and emotional symptoms matter regardless of impact, just as physical symptoms require attention to enable efficient diagnosis and treatment. ${ }^{36}$ Challenging this view, however, is evidence that even when symptom scores are taken into consideration, impact scores predict key outcomes such as contact with psychiatric services. ${ }^{37}$ The SDQ impact scale amalgamates three components: distress and impaired function for the child, and perceived family burden, each of which may have different significance for parental wellbeing. Adding further complexity to the picture, impact scores differ in salience across psychiatric symptoms: some symptoms (e.g. a fear of heights) are, in most circumstances, completely manageable; in contrast, other symptoms (e.g. low mood, poor sleep) are themselves markers of impact, such that a global impact score for an individual with a variety of different symptoms can be difficult to interpret. Our finding that impact scores predicted parental well-being echoes findings related to cognitive impairment at the other end of the lifespan: well-being in carers of individuals with dementia is often more closely related to the behavioural or emotional impact of dementia than to the loss of cognitive function per se. ${ }^{38}$ Together, these findings suggest that changes in impact scores, although complex, are a potentially valuable marker of intervention success, providing an index of the extent to which initiatives aimed at the affected individual have positive ripple effects on family members. Further studies are required to examine how parents' appraisals or coping strategies intervene between child characteristics and perceived impact, and the extent to which perceived impact could itself be a marker of parental well-being or an intervention target.

\section{Parental well-being is influenced by genetic diagnosis of their child's intellectual disability}

The genetic diagnosis underlying childhood intellectual disability made a significant direct contribution to impact, with parents of children with $\mathrm{CNV}$-associated intellectual disability reporting 


\begin{tabular}{|c|c|c|c|c|c|c|c|c|c|c|c|c|}
\hline & \multicolumn{4}{|c|}{ Perceived impact } & \multicolumn{4}{|c|}{ Caregiver depressive symptoms } & \multicolumn{4}{|c|}{$\begin{array}{c}\text { Indirect effects on caregiver } \\
\text { depressive symptoms via perceived } \\
\text { impact }\end{array}$} \\
\hline & Est. & s.e. & $\beta$ & $P$ & Est. & s.e. & $\beta$ & $P$ & Est. & s.e. & $\beta$ & $P$ \\
\hline \multicolumn{13}{|l|}{ Child characteristics } \\
\hline Age & 0.072 & 0.040 & 0.072 & & 0.180 & 0.120 & 0.064 & & & & & \\
\hline Gender & -0.088 & 0.241 & -0.015 & & 0.105 & 0.699 & 0.007 & & & & & \\
\hline Developmental quotient & -0.005 & 0.008 & -0.036 & & 0.005 & 0.022 & 0.012 & & & & & \\
\hline Physical disability & 0.176 & 0.091 & 0.094 & & 0.247 & 0.244 & 0.048 & & & & & \\
\hline DAWBA ASD & 1.090 & 0.304 & 0.159 & $\star * *$ & 0.398 & 1.037 & 0.021 & & & & & \\
\hline DAWBA CD/ODD & 1.950 & 0.260 & 0.289 & $\star * *$ & 1.792 & 0.980 & 0.096 & & & & & \\
\hline DAWBA other & 1.302 & 0.246 & 0.209 & $* * *$ & -0.453 & 0.857 & -0.026 & & & & & \\
\hline Perceived impact & & & & & 0.706 & 0.135 & 0.255 & $* * *$ & & & & \\
\hline \multicolumn{13}{|l|}{ CNV characteristics } \\
\hline De novo/inherited & -0.025 & 0.319 & -0.004 & & 0.824 & 0.899 & 0.050 & & -0.018 & 0.225 & -0.001 & \\
\hline De novo/unknown & -0.044 & 0.279 & -0.008 & & -0.747 & 0.866 & -0.048 & & -0.031 & 0.197 & -0.002 & \\
\hline Deletion/duplication & 0.296 & 0.248 & 0.051 & & -0.302 & 0.723 & -0.019 & & 0.209 & 0.179 & 0.013 & \\
\hline Deletion/other & -0.791 & 0.551 & -0.054 & & -1.524 & 1.728 & -0.037 & & -0.558 & 0.405 & -0.014 & \\
\hline CNV size (bp) & -0.160 & 0.103 & -0.058 & & -0.295 & 0.328 & -0.038 & & -0.113 & 0.077 & -0.015 & \\
\hline \multicolumn{13}{|l|}{ Social circumstances } \\
\hline Life events & 0.006 & 0.110 & 0.002 & & 0.764 & 0.312 & 0.105 & * & & & & \\
\hline IMD & -0.033 & 0.043 & -0.032 & & 0.039 & 0.128 & 0.014 & & & & & \\
\hline Number of children & 0.032 & 0.273 & 0.005 & & 0.056 & 0.795 & 0.003 & & & & & \\
\hline Number of adults & 0.748 & 0.859 & 0.051 & & 2.176 & 1.600 & 0.053 & & & & & \\
\hline Solo parent & -0.077 & 0.350 & -0.011 & & 0.384 & 0.935 & 0.019 & & & & & \\
\hline
\end{tabular}

higher perceived impact, indirectly influencing caregiver distress. Neither variant inheritance nor postcode-level socioeconomic factors explained the contrast in well-being between CNV and other diagnostic groups. One plausible explanation for the influence of genetic diagnosis on parental distress could be the elevated proportion of the CNV group (versus the remainder of this sample) who received a DAWBA likelihood diagnosis of either oppositional defiant disorder or conduct disorder. Strengthening the view that psychiatric phenotypes may contribute to impact, detailed face-toface characterisation of a subset of individuals from the IMAGINE-ID cohort with specific recurrent CNV diagnoses ${ }^{22}$ found extremely high levels of psychopathology and comorbidity. Hence, persistent complex emotional-behavioural difficulties for the CNV group may exert an especially negative long-term effect on parents and the wider family. However, in the current analysis, the effects of CNV diagnosis on impact and distress were found to be independent of phenotypic variations across the sample, suggesting that the association between $\mathrm{CNV}$ diagnosis and impact may reflect not the direct consequences of aetiology on phenotype, but rather between-group differences in the diagnostic journey of children and families in this cohort. Beyond diagnosis, the extent of support available to children and families may differ between groups: the SNV group on average have higher physical disability scores and more severe intellectual disability, which may result in a more comprehensive and sustained package of educational, medical and social support, with a consequential influence on parental appraisal of impact. Here, the literature on violations of parental expectations with regards to the impact of premature birth ${ }^{39}$ may provide a useful analogy. That is, if the CNV group is often characterized by mild-to-moderate intellectual disability coupled with prominent behavioural problems, parents in this group are likely to face both greater violations of expectations and reduced tolerance from the community, leading to greater problems of uncertainty and social isolation. Further work both within IMAGINE-ID and other cohorts is needed to tease apart these alternative proposals.

\section{Limitations}

This study did not include children with intellectual disability of unknown origin, or intellectual disability of acquired origin; these comparison groups would provide a broader context for understanding the role of aetiology in parental experience. The study applied extremely broad categorisation of genetic diagnoses, insensitive to the distinct spatiotemporal expression patterns, molecular mechanisms, biological pathways, and polygenic and environmental interactions of each individual's rare disorder. Several potential sources of ascertainment bias deserve mention. First, the number of eligible families who received information about the study but did not consent or participate is not known, hence we cannot estimate a response rate or assess factors influencing participation. Second, participants from deprived areas were underrepresented in the cohort. This may be explained by socioeconomic status (SES)-related-related differences in access to genetic testing, or in the likelihood of volunteering for this research study and completing data entry. A further possibility is that SES predicts the likelihood of a genetic diagnosis underlying intellectual disability: deprivation shifts the balance between mild and severe intellectual disability ${ }^{40}$ and may thus be associated with higher likelihood of multifactorial or acquired causes. Nevertheless, the sample was sufficiently diverse to test for association between parental well-being and neighbourhood characteristics and, echoing results from previous studies, ${ }^{4,7}$ we did not find any such association. That is, we found no evidence that SES confounds the effect of genetic diagnosis on parental impact and well-being.

Several limitations in data collection also deserve note. Betweenstudy differences in the definition and measurement of distress among parents caring for children with intellectual disability precluded comparison of effect sizes across studies. Online data collection precluded psychometric assessment to objectively confirm the presence of intellectual disability and its severity in the diagnosed child. A total of $22 \%$ of respondents opted to complete assessments with telephone support or via face-to-face interview, which may influence self-report via social desirability effects. ${ }^{41}$ The study did 
not measure household-level poverty, in particular household income, material hardship and receipt of welfare benefits, which limited our ability to investigate socioeconomic contributors to parental well-being. In addition, we had no measure of parental physical health, an important predictor of mental health in previous studies, ${ }^{7,8}$ and potentially of heightened significance for parents with inherited genomic variants. Further, the limited number of fathers involved as primary respondents in our study made it difficult to interpret the observed informant effects, namely a weaker contrast in well-being between IMAGINE-ID parents and general population when the primary respondent was a father. Note also that reliance on proxy ratings for partners limited the independence of measures, and ability to investigate convergence or divergence between parents. For these reasons, future studies should seek to gather information directly from more than one parent/carer in each family. The current study design is cross-sectional, and future longitudinal work is needed to clarify the relations between impact and distress, establish a causal pathway integrating genetic, developmental and family factors, and identify modifiable factors that could be effective intervention targets.

In summary, this study confirms the high risk of emotional distress associated with caring for a child with intellectual disability. Our findings are consistent with previous studies showing that children's physical and behavioural characteristics and parental appraisal of impact contribute to variation in parents' well-being. A novel contribution is the finding that genetic diagnosis type exerts an additional influence. Further work is needed to elucidate the mechanisms underlying this association; these are likely to be complex, reflecting not only intellectual disability phenotype, but also the evolving narrative of parental experiences both within and outside the family. Given rapid increases in access to genetic testing and diagnostic yields, understanding how genetic diagnoses may be associated with parental mental health is clinically important. Future research should address the trajectories of parental experience before, at and after receiving a genetic diagnosis for their child, to determine optimal timings of family risk assessment and interventions to promote long-term well-being of individuals and families.

Kate Baker (1D, MBBS PhD, Programme Leader Track and Honorary Consultant in Clinica Genetics, MRC Cognition and Brain Sciences Unit, University of Cambridge, UK;

Rory T. Devine (D), PhD, Lecturer in Developmental Psychology, School of Psychology, University of Birmingham, UK; Elise Ng-Cordell, MA, Research Assistant, MRC Cognition and Brain Sciences Unit, University of Cambridge, UK; F. Lucy Raymond, MBBS DPhil FRCP, Professor of Medical Genetics and Neurodevelopment, Cambridge Institute for Medical Research, University of Cambridge, UK; IMAGINE-ID consortium, see Supplementary material for membership and affiliations; Claire Hughes, PhD, Professor and Deputy Director, Centre for Family Research, Department of Psychology, University of Cambridge, UK

Correspondence: Kate Baker. Email: kate.baker@mrc-cbu.cam.ac.uk

First received 13 Aug 2019, final revision 14 Nov 2019, accepted 20 Dec 2019

\section{Supplementary material}

Supplementary material is available online at https://doi.org/10.1192/bjp.2020.38.

\section{Funding}

This work was supported by the UK Medical Research Council (grant number G101400 to K.B.), UK Medical Research Council and Medical Research Foundation (grant number MR-N022572-1 to the IMAGINE-ID study; Principle Investigators: David H. Skuse, F Lucy Raymond, Jeremy Hall, Marianne Van den Bree, Michael J. Hall) and the Baily Thomas Charitable Trust (to K.B.).

\section{Acknowledgements}

We thank the IMAGINE-ID study families, as well as all IMAGINE-ID research scientists, study and data managers, and clinical and administrative staff who make the study possible. Data entry of household structure variables was carried out by Annabel Holmes and Sophie Frear.

\section{Data availability}

Authors' access to the study data was permitted via a data access agreement. The data associated with the manuscript is not currently publicly available.

\section{Author contributions}

K.B. R.T.D. and C.H. formulated the research questions, designed and carried out the analysis and wrote the article. The IMAGINE-ID consortium designed the wider study, recruited participants, and collected and processed the data. E.N.-C. and F.L.R. reviewed and amended the manuscript.

\section{References}

1 Families Special Interest Research Group of IASSIDD. Families supporting a child with intellectual or developmental disabilities: the current state of knowledge. J Appl Res Intellect Disabil 2014; 27(5): 420-30.

2 Rare Disease UK. Living with a Rare Condition: The Effect on Mental Health 2018. Rare Disease UK, 2018 (https://www.raredisease.org.uk/our-work/living-with-a-rare-condition-the-effect-on-mental-health-2018/).

3 Olsson MB, Hwang CP. Depression in mothers and fathers of children with intellectual disability. J Intellect Disabil Res 2001; 45(Pt 6): 535-43.

4 Emerson E. Mothers of children and adolescents with intellectual disability: social and economic situation, mental health status, and the self-assessed social and psychological impact of the child's difficulties. J Intellect Disabil Res 2003; 47(Pt 4-5): 385-99.

5 Emerson E, McCulloch A, Graham H, Blacher J, Llwellyn GM, Hatton C. Socioeconomic circumstances and risk of psychiatric disorders among parents of children with early cognitive delay. Am J Intellect Dev Disabil 2010; 115(1): 30-42.

6 McConnell JS, Savage A. Stress and resilience among families caring for children with intellectual disability: expanding the research agenda. Curr Dev Disord Rep 2015; 2: 100-9.

7 Emerson E, Hatton C, Llewellyn G, Blacher J, Graham H. Socio-economic position, household composition, health status and indicators of the well-being of mothers of children with and without intellectual disabilities. J Intellect Disabil Res 2006; 50(Pt 12): 862-73.

8 Olsson MB, Hwang CP. Socioeconomic and psychological variables as risk and protective factors for parental well-being in families of children with intellectual disabilities. J Intellect Disabil Res 2008; 52(12): 1102-13.

9 Gallagher S, Pilch M, Hannigan A. Prior depressive symptoms and persistent child problem behaviours predict future depression in parents of children with developmental disabilities: the growing up in Ireland cohort study. Res Dev Disabil 2018; 80: 170-9.

10 Baker BL, Blacher J, Olsson MB. Preschool children with and without developmental delay: behaviour problems, parents' optimism and well-being. J Intellect Disabil Res 2005; 49(Pt 8): 575-90.

11 Oelofsen N, Richardson P. Sense of coherence and parenting stress in mothers and fathers of preschool children with developmental disability. J Intellect Dev Disabil 2006; 31(1): 1-12.

12 Bolourian Y, Blacher J. Comorbid behavior problems among youth with intellectual and developmental disabilities: a developmental focus. Int Rev Res Dev Disabil 2018; 55: 181-212.

13 Cohen SR, Zeedyk SM, Tipton LA, Rodas NV, Blacher J. Fathers of children with or without ID: understanding long-term psychological symptoms. J Intellect Disabil Res 2016; 60(4): 295-307.

14 Woodman AC, Mawdsley HP, Hauser-Cram P. Parenting stress and child behavior problems within families of children with developmental disabilities: transactional relations across 15 years. Res Dev Disabil 2015; 36C: 264-76.

15 Gilissen C, Hehir-Kwa JY, Thung DT, van de Vorst M, van Bon BW, Willemsen $\mathrm{MH}$, et al. Genome sequencing identifies major causes of severe intellectual disability. Nature 2014; 511(7509): 344-7.

16 Wright CF, FitzPatrick DR, Firth HV. Paediatric genomics: diagnosing rare disease in children. Nat Rev Genet 2018; 19(5): 325.

17 Hodapp RM, Dykens EM. Genetic disorders of intellectual disability: expanding our concepts of phenotypes and of family outcomes. J Genet Couns 2012; 21 (6): 761-9.

18 Griffith GM, Hastings RP, Nash S, Hill C. Using matched groups to explore child behavior problems and maternal well-being in children with Down syndrome and autism. J Autism Dev Disord 2010; 40(5): 610-9.

19 Hall SS, Burns DD, Reiss AL. Modeling family dynamics in children with fragile $x$ syndrome. J Abnorm Child Psychol 2007; 35(1): 29-42. 
20 Adams D, Clarke S, Griffith G, Howlin P, Moss J, Petty J, et al. Mental health and well-being in mothers of children with rare genetic syndromes showing chronic challenging behavior: a cross-sectional and longitudinal study. Am J Intellect Dev Disabil 2018; 123(3): 241-53.

21 Girirajan S, Rosenfeld JA, Coe BP, Parikh S, Friedman N, Goldstein A, et al. Phenotypic heterogeneity of genomic disorders and rare copy-number variants. N Engl J Med 2012; 367(14): 1321-31.

22 Chawner S, Owen MJ, Holmans P, Raymond FL, Skuse D, Hall J, et al. Genotypephenotype associations in children with copy number variants associated with high neuropsychiatric risk in the UK (IMAGINE-ID): a case-control cohort study. Lancet Psychiatry 2019; 6(6): 493-505.

23 Uher R, Goodman R. The Everyday Feeling Questionnaire: the structure and validation of a measure of general psychological well-being and distress. Soc Psychiatry Psychiatr Epidemiol 2010; 45(3): 413-23.

24 Goodman R. Psychometric properties of the Strengths and Difficulties Questionnaire. J Am Acad Child Adolesc Psychiatry 2001; 40(11): 1337-45

25 Goodman R, Ford T, Richards H, Gatward R, Meltzer H. The Development and Well-Being Assessment: description and initial validation of an integrated assessment of child and adolescent psychopathology. J Child Psychol Psychiatry 2000; 41(5): 645-55.

26 Emerson E, Hatton C. Mental health of children and adolescents with intellectual disabilities in Britain. Br J Psychiatry 2007: 191: 493-9.

27 Ford $\mathrm{T}$, Goodman $\mathrm{R}$, Meltzer $\mathrm{H}$. The relative importance of child, family, school and neighbourhood correlates of childhood psychiatric disorder. SOC Psychiatry Psychiatr Epidemiol 2004; 39(6): 487-96.

28 McEwen FS, Stewart CS, Colvert E, Woodhouse E, Curran S, Gillan N, et al. Diagnosing autism spectrum disorder in community settings using the Development and Well-Being Assessment: validation in a UK population-based twin sample. J Child Psychol Psychiatry 2016; 57(2): 161-70.

29 Smith T. The English Indices of Deprivation - Technical Report. Department for Communities and Local Government, 2015.

30 Brugha T, Bebbington $\mathrm{P}$, Tennant $\mathrm{C}$, Hurry J. The List of Threatening Experiences: a subset of 12 life event categories with considerable long-term contextual threat. Psychol Med 1985; 15(1): 189-94.
31 Office for National Statistics. Families and Households Statistics Explained. ONS, 2019 (https://www.ons.gov.uk/peoplepopulationandcommunity/birth sdeathsandmarriages/families/articles/familiesandhouseholdsstatisticsexplained/2019-08-07)

32 Muthén LK, Muthén BO. Mplus User's Guide, Eighth Edition. Muthén \& Muthén, 2017 (https://www.statmodel.com/download/usersguide/MplusUserGuide Ver 8.pdf).

33 Kline RB. Principles and Practice of Structural Equation Modeling. Guilford Press, 2011.

34 Brown TA. Confirmatory Factor Analysis for Applied Research (2nd ed). Guilford Press, 2015.

35 UK Office of National Statistics. Mental health of children and young people in Great Britain, 2004. NHS Digital, 2005 (https://digital.nhs.uk/data-and-information/publications/statistical/mental-health-of-children-and-young-peoplein-great-britain/mental-health-of-children-and-young-people-in-great-britain2004)

36 Rutter M. Research review: child psychiatric diagnosis and classification: concepts, findings, challenges and potential. J Child Psychol Psychiatry 2011; 52(6): 647-60.

37 Stringaris A, Goodman R. The value of measuring impact alongside symptoms in children and adolescents: a longitudinal assessment in a community sample. J Abnorm Child Psychol 2013; 41(7): 1109-20.

38 Waite A, Bebbington P, Skelton-Robinson M, Orrell M. Social factors and depression in carers of people with dementia. Int J Geriatr Psychiatry 2004; 19(6): 582-7.

39 Macey TJ, Harmon RJ, Easterbrooks MA. Impact of premature birth on the development of the infant in the family. J Consult Clin Psychol 1987; 55(6): 846-52.

40 Emerson E. Deprivation, ethnicity and the prevalence of intellectual and developmental disabilities. J Epidemiol Community Health 2012; 66(3): 218-24.

41 Zhang X, Kuchinke L, Woud ML, Velten J, Margraf J. Survey method matters: online/offline questionnaires and face-to-face or telephone interviews differ. Comput Hum Behav 2017; 71: 172-80. 